\title{
¿Cuál es la formación de la planta académica de la UNAM? El caso de los profesores de asignatura
}

\author{
Ruth Torres Carrasco
}

\section{Resumen}

Este texto presenta una breve descripción estadística de una de las agrupaciones académicas más importantes de la unAm, el profesorado de asignatura. Muestra de manera genérica cuál es su formación, edades y antigüedad de quienes desempeñan una de las funciones sustantivas de la Universidad: la docencia. La información que se expone toma como base los subsistemas universitarios donde se agrupa la mayor cantidad de profesores con esta figura académica: escuelas y facultades, entidades multidisciplinarias, la Escuela Nacional Preparatoria (ENP) y el Colegio de Ciencias y Humanidades (CCH).

Palabras clave: profesores de asignatura, Escuela Nacional Preparatoria (ENP), Colegio de Ciencias y Humanidades (CCH), UNAM.

\section{WHICH IS THE TRAINING OF tHE ACADEMIC StAFF AT UNAM? THE ADJUNCT FAC- ULTY CASE}

\section{Abstract}

This article presents a brief statistical description of one of the most important academic groups at the National Autonomous University of Mexico (UNAm): the adjunct faculty. It shows, in general, which is the formation, age and seniority of those who play one of the university key roles: teaching. This information takes into account the university subsystem in which we find most of the teachers characterize as adjuncts: schools and faculties, multidisciplinary centers, National Preparatory High School (ENP) and College of Sciences and Humanities ( $\mathrm{CCH}$ ).

Keywords: adjunct faculty, National Preparatory High School (ENP), College of Sciences and Humanities $(\mathrm{CCH})$, UNAM. 


\section{Ruth Torres Carrasco}

rtorresc@unam.mx

Doctora en Educación con especialidad en Formación Inicial y Permanente de Profesionales de la Educación y la Innovación Educativa, por la Facultad de Educación de la Universidad Complutense de Madrid, España. Licenciada y Maestra en Administración, con mención honorífica; egresada del Diplomado en Docencia Universitaria (en dos ocasiones) por la Facultad de Contaduría Administración (FCA), de la Universidad Nacional Autónoma de México (UNAM).

Actualmente, se desempeña como Subdirectora de Desarrollo Académico, en la Coordinación de Desarrollo Educativo e Innovación Curricular (CODEIC) de la unAm. En la academia desarrolla actividades como profesora Titular de Tiempo Completo Definitiva por oposición en el área de Recursos Humanos, participa como docente de nivel licenciatura, maestría y doctorado del Programa de Posgrado en Ciencias de la Administración y del Programa Maestría y Doctorado en Educación en Ciencias de la Salud, ambos de la unam. Profesora invitada en las áreas de Administración y Educación a nivel nacional e internacional.

${ }^{1}$ La unam es considerada el proyecto educativo más conocido e importante de

México. De acuerdo con

su portal de estadística universitaria, durante el ciclo escolar 2018-2019 atendió en sus recintos y aulas a un total de 356,530 alumnos; de éstos 30,089 fueron de nivel posgrado; 213,004 de licenciatura; 112,588

de bachillerato y 849 de los niveles técnico o propedéutico que se imparten en la Universidad.

\section{Introducción}

De acuerdo con el Diccionario de la lengua española, docencia se define como la práctica y ejercicio del docente, esta palabra proviene del latín docēre que significa enseñar. Así pues, docencia se puede señalar como una actividad realizada por aquella persona que enseña o que está involucrada con la enseñanza. Otras formas de designar al docente son como profesor, maestro, catedrático, educador, asesor o incluso enseñante; todas estas significaciones están asociadas a lo que Prellezo define como "los titulares de la función docente" (Diccionario de Ciencias de la Educación, 2009).

En el caso de la Universidad Nacional Autónoma de México (UNAM), la función docente recae en un colectivo de personas que, en los diversos niveles de formación, promueven la adquisición de conocimientos, habilidades, actitudes y valores para egresar bachilleres y profesionistas; profesores, técnicos e investigadores. ${ }^{1}$ Para formar a los estudiantes y de acuerdo con el Registro Único del Personal Académico (RUPA) de la Dirección General de Asuntos del Personal Académico (DGAPA) de la unAm, la institución contaba con 41,770 académicos hasta el pasado mes de junio de 2019.

Para entender mejor la dinámica de la función docente y de las personas que la llevan a cabo, presentaremos una caracterización muy general de la composición de los académicos universitarios, con la intención de dar cuenta de la diversidad de quienes tienen a su cargo una de las funciones principales de la institución, y que, además, representa en términos económicos 61.2\% del presupuesto financiero de toda la Universidad, la docencia. 
El porcentaje más alto lo representa la categoría o el nombramiento de profesor de asignatura con 63\% del total, 30\% se desempeña en la denominada categoría de profesor o investigador de carrera de tiempo completo o jornada completa; mientras que el restante $7 \%$ se distribuye en otro tipo de designaciones que pueden ser ayudantes o técnicos e incluso una combinación de nombramientos por hora y de carrera.

Para este texto nos centraremos en describir a los docentes de asignatura con el propósito de caracterizar a ese colectivo, en el que, como puede observarse, se concentra el mayor número de personas que conforma a la comunidad académica universitaria y cuya principal actividad es la labor docente, la tarea de enseñar. En la gráfica 1 se muestra la distribución porcentual de las grandes agrupaciones de los profesores universitarios.

\section{Profesores por nombramiento}

Gráfica 1. Profesores de la UnAM por nombramiento. Elaboración propia, con datos de RUPA-DGAPA, junio 2019.

Gráfica 2. Profesores de asignatura de la UNAM por entidad académica. Elaboración propia, con datos de RUPA-DGAPA, junio 2019.

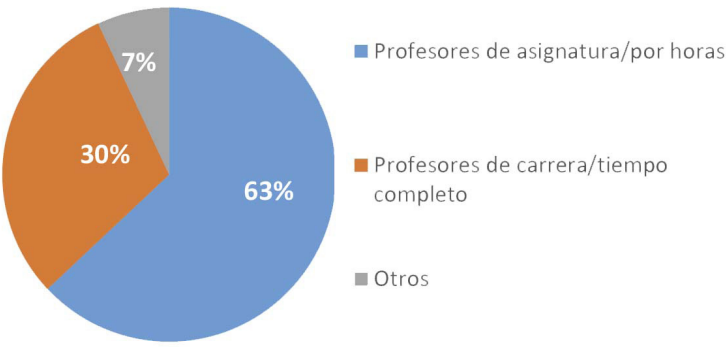

Es importante destacar que, de los profesores de asignatura, 15,002 efectuaron su tarea formativa en alguna escuela o facultad; 7,567 lo hicieron en alguna entidad multidisciplinaria; 2,839 en el Colegio de Ciencias y Humanidades (CCH); mientras que 1,801 la realizaron en alguno de los planteles de la Escuela Nacional Preparatoria (ENP). En gráfica 2 se muestra el porcentaje por entidades. Puede observarse que la mayor cantidad de profesores de asignatura realizan su labor docente en alguna escuela o facultad de la Universidad. A lo largo del artículo se hablará de cada una de estas entidades académicas y el tipo de formación que presenta su plantilla docente.

\section{Profesores de asignatura por entidades académicas}

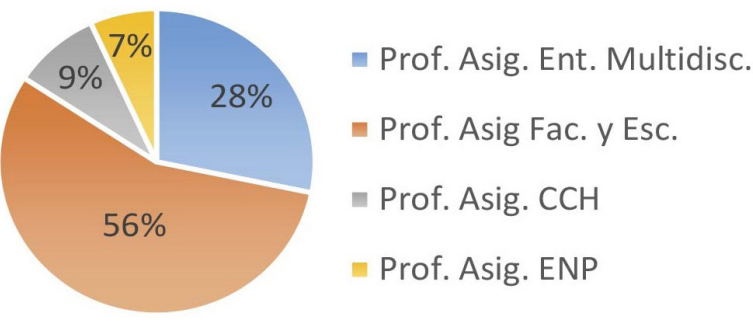


Gráfica 3. Profesores de asignatura de la unAm por sexo en escuelas y facultades. Elaboración propia, con datos de RUPA-DGAPA, junio 2019.

Gráfica 4. Profesores de asignatura de la unam por edad y antigüedad en escuelas y facultades. Elaboración propia, con datos de RUPA-DGAPA, junio 2019.

\section{Los profesores de asignatura en escuelas y facultades}

Iniciaremos el análisis con la mayor agrupación de profesores de asignatura en la unAM, quienes realizan su tarea de formar estudiantes en alguna de las quince facultades o nueve escuelas nacionales. De acuerdo con la base de datos RUPA, en el mes de junio de 2019, en estas entidades académicas 15,002 personas se desempeñaron como profesores de asignatura. Su distribución por sexo, edad y antigüiedad se muestran en las gráficas 3 y 4.

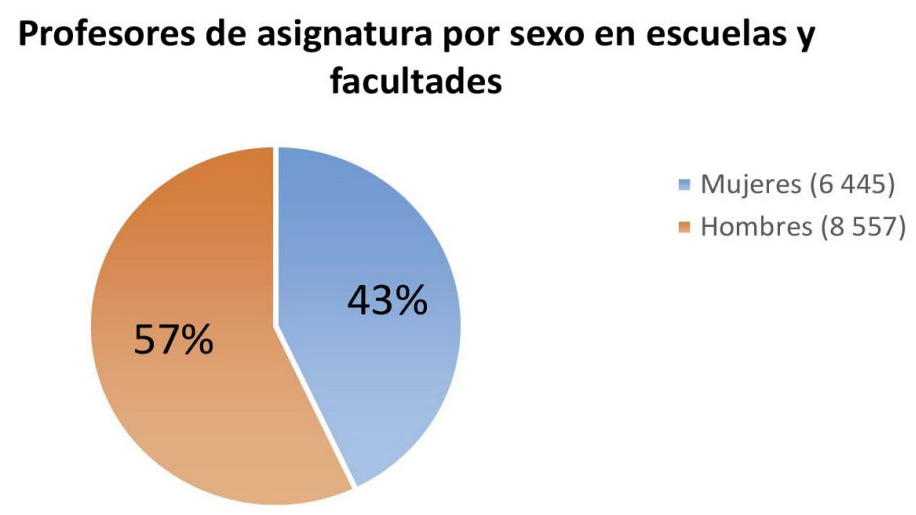

\section{Edad y antigüedad promedio de los profesores de asignatura en escuelas y facultades}

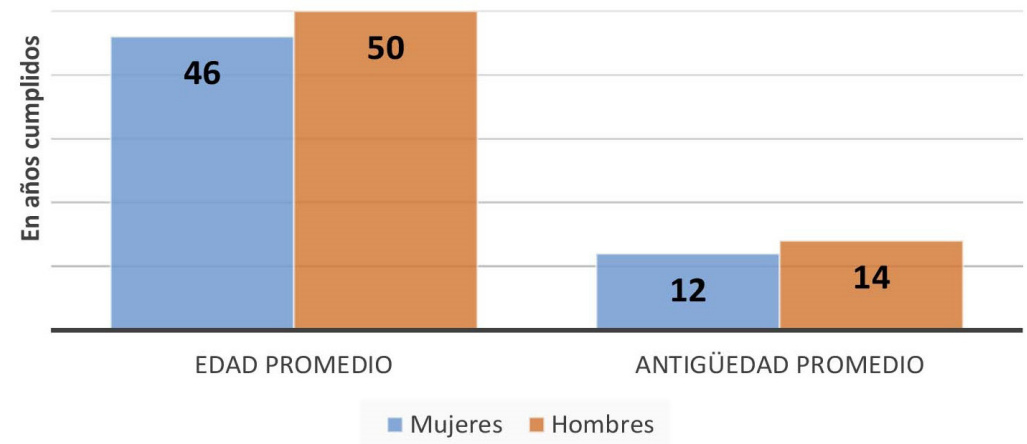

El nivel de estudios que poseen los docentes de asignatura en las escuelas y facultades de la unAm es un dato de suma importancia, ya que esto permite el desarrollo de una docencia donde se garantice que los estudiantes reciban conocimientos sólidos, actuales y vinculados con las necesidades sociales y con la práctica profesional de los diversos campos disciplinares. De acuerdo con la información que se ha rescatado, de los 15,002 profesores de asignatura en estas entidades académicas 17 poseen posdoctorado; 2,043, el título de doctor; 1,878, el de especialista; 4,187, el de maestría; 6,155, el de licenciatura y 139 son pasantes, 12 tienen algún diploma de técnico, mientras que de 571 personas que poseen el nombramiento de profesor de asignatura, no se dispone de información sobre su grado académico. En la gráfica 5 se muestra la distribución 
Gráfica 5. Profesores de asignatura de la unAm por nivel de estudios en escuelas y facultades.

Elaboración propia, con datos de RUPA-DGAPA, junio 2019.
Gráfica 6. Nivel de estudio de profesores de asignatura de la unam por sexo en escuelas y facultades. Elaboración propia, con datos de RUPA-DGAPA, junio 2019. porcentual del nivel de estudios de los profesores de asignatura en escuelas y facultades de la unam.

\section{Nivel de estudios de los profesores de asignatura en escuelas y facultades de la UNAM}

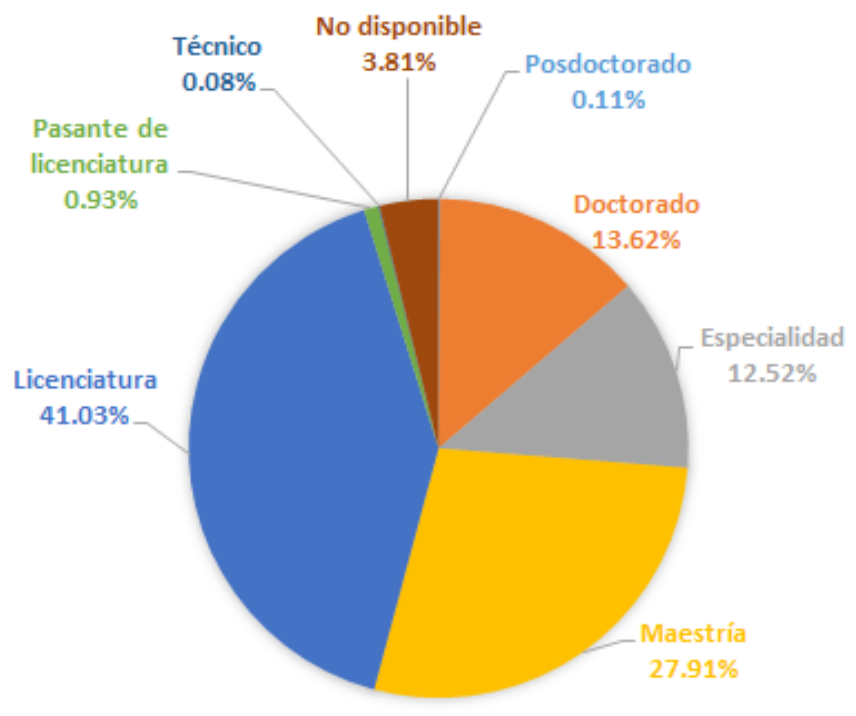

Los profesores de asignatura en las escuelas y facultades representan un importante número de personas a quienes pocas veces se les estudia con la finalidad de conocer cuáles son sus características, su formación o grado de estudios, sus edades o el tiempo que tienen desempeñando la tarea formativa. Sin embargo, en los últimos años se ha privilegiado la contratación de profesores de asignatura con algún estudio de posgrado, pues "la educación de posgrado, constituye el conjunto de procesos de enseñanza-aprendizaje dirigidos a garantizar la preparación de los graduados universitarios" (Manzo Rodríguez, Rivera Michelena y Rodríguez Orozco, 2006), lo que además representa uno de los tantos elementos a valorar en los procesos de acreditación o certificación de la educación universitaria, de ahí la importancia de conocer a este colectivo.

Nivel de estudios de los profesores de asignatura por sexo en escuelas y facultades

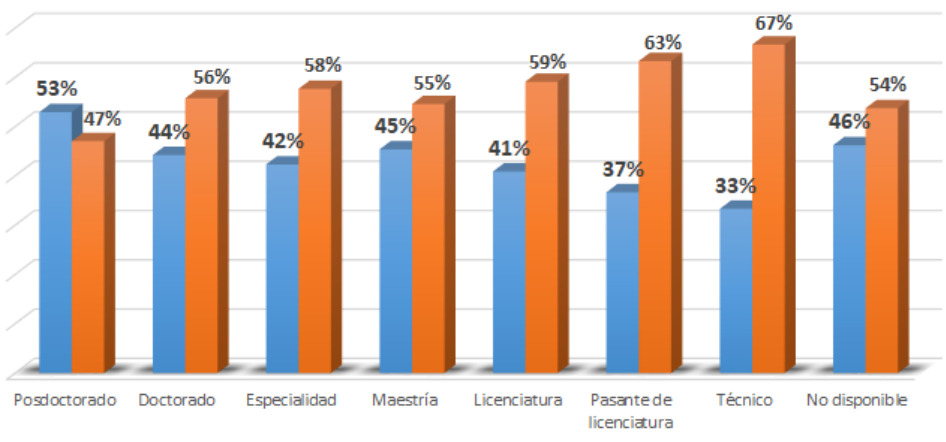

- Mujeres n Hombres 


\section{Los profesores de asignatura en las entidades multidisciplinares}

La masificación de la educación superior en los años setenta y la demanda por abrir más espacios en las instituciones de educación superior trajeron como consecuencia que la unAm no pudiera recibir en su campus principal de Ciudad Universitaria a más estudiantes para su preparación en el nivel licenciatura. Para entonces, un plan de crecimiento fue ideado para atender la demanda de espacios para la formación superior y, en particular, para atender la demanda en la zona conurbada a la Ciudad de México. Con esta estrategia de expansión se crearon las Ilamadas Escuelas Nacionales de Estudios Profesionales (ENEP) las cuales, con el paso del tiempo, se convirtieron en Facultades de Estudios Superiores (FES), a partir del desarrollo y creación de programas de posgrado, permitiendo otorgar los grados de maestría y doctorado en los ámbitos disciplinares que atienden. En estas sedes académicas, que en total suman cinco, la multidisciplina, así como la combinación de las distintas áreas del conocimiento son la característica principal, por lo que la riqueza académica toma diversas formas a través del trabajo de los docentes.

Siguiendo con la misma estructura de análisis presentada para el profesorado de asignatura en escuelas y facultades, en las unidades multidisciplinarias se cuenta con un total de 7,567 profesores de asignatura, lo que representa $28 \%$ del total de los docentes con esta categoría en toda la UNAM (véase gráfica 2). Así, se ubica como el segundo colectivo docente más grande después del conjunto de personas en las escuelas y facultades. En las gráficas 7 y 8 se muestran los porcentajes por sexo, así como la distribución por antigüedades y edades de esta agrupación.

Gráfica 7. Sexo de los profesores de asignatura en entidades multidisciplinarias de la unam. Elaboración propia, con datos de RUPA-DGAPA, junio 2019

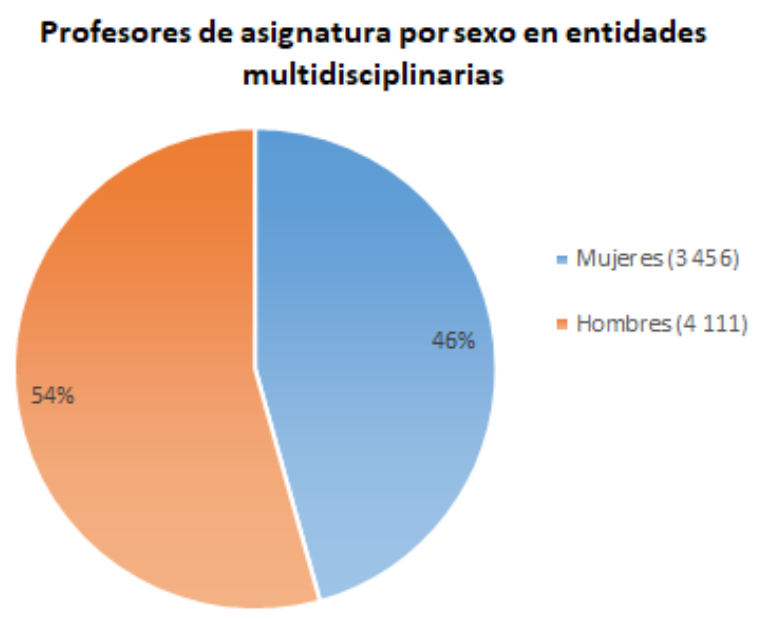

El nivel de escolaridad que poseen los profesores de asignatura en las entidades multidisciplinarias es una información que da cuenta de la diversidad formativa que hay en estos recintos universitarios. Así, tenemos una composición diversa en la que se cuenta con cuatro académicos con posdoctorado, 521 con doctorado, 1,901 con maestría, 3,799 con licenciatura, 860 poseen el grado de 
Gráfica 8. Distribución por antigüedad y edad de los profesores de asignatura en entidades multidisciplinarias de la unAm. Elaboración propia, con datos de RUPA-DGAPA, junio 2019.

Gráfica 9. Nivel de estudios de los profesores de asignatura en entidades multidisciplinarias de la unam. Elaboración propia, con datos de RUPA-DGAPA, junio 2019 especialistas, 12 tienen alguna preparación de nivel técnico, 101 son pasantes de alguna licenciatura, mientras que de 369 personas que ostentan un nombramiento de profesor de asignatura no se tiene disponible información o bien no poseen algún grado de estudios. En la gráfica 9 se presenta la distribución porcentual por grado de estudios.

\section{Edad y antigüedad promedio de los profesores de} asignatura en entidades multidisciplinarias
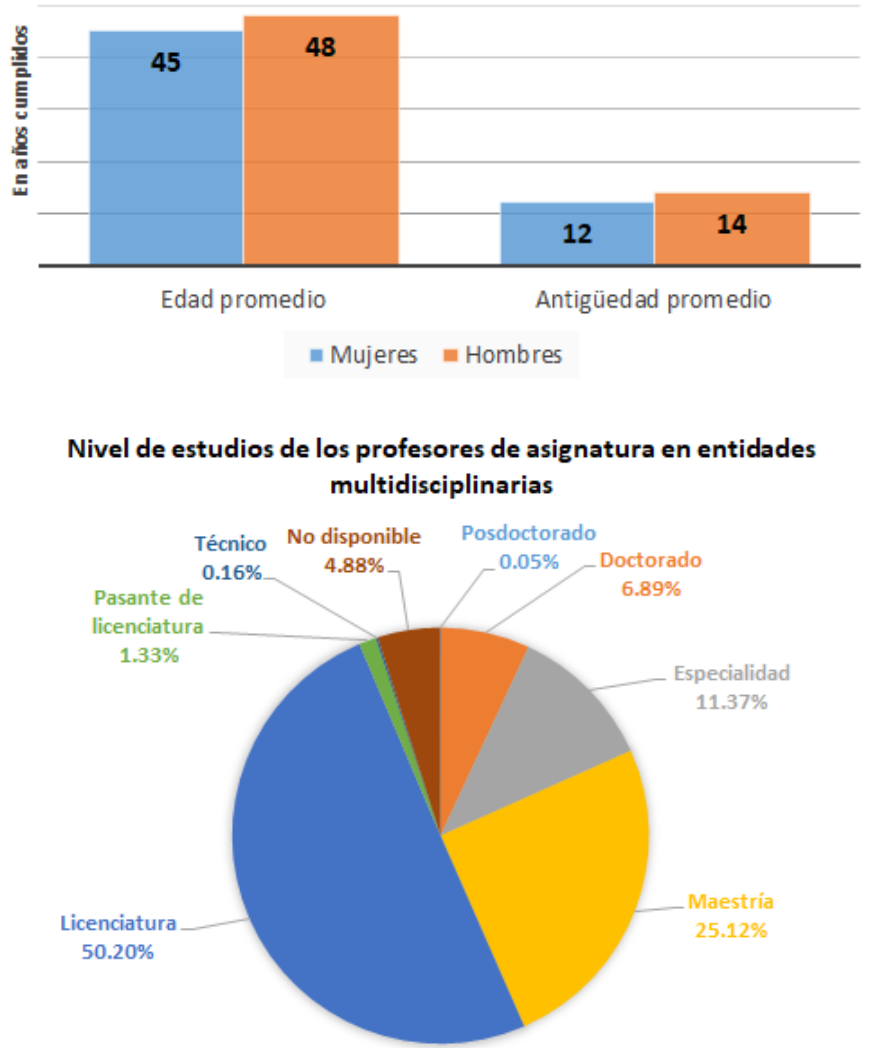

Es importante mencionar que los profesores de asignatura en las cinco entidades multidisciplinarias -aun cuando en número son menos que en las facultades y escuelas- atienden a una población estudiantil en 61 licenciaturas; mientras que los profesores de asignatura forman estudiantes en 75 licenciaturas en 18 facultades o escuelas (Martínez Hernández y colaboradores, 2019), lo que da cuenta que las entidades multidisciplinarias para lograr sus objetivos formativos se apoyan en este importante colectivo docente.

\section{Los profesores de asignatura en la Escuela Nacional Preparatoria}

En 1867 es fundada por decreto presidencial la Escuela Nacional Preparatoria, misma que se crea como un espacio educativo que se constituyó como base 
Gráfica 10. Sexo de los profesores de asignatura de la ENP de la unAm. Elaboración propia, con datos de RUPA-DGAPA, junio 2019. para acceder a las entonces llamadas Escuelas de Altos Estudios (Dirección General de la Escuela Nacional Preparatoria, s.f.). Para 1945, se publica la Ley Orgánica de unAm, reconfigurándose la vida académica y con ello los estudios preparatorios, que permiten formar a los bachilleres, quienes al concluir sus estudios podrán ingresar a alguna escuela o facultad para una preparación a nivel licenciatura. Durante la década de los cincuenta, la ENP inicia su crecimiento en espacio e infraestructura construyéndose una serie de planteles en toda la ciudad que extendieron su proyecto educativo. Actualmente, se cuentan con nueve planteles donde los docentes adscritos a este subsistema universitario atienden a poco más de cincuenta mil estudiantes de acuerdo con datos de la Agenda Estadística de la unAm 2019 (UnAM, s.f.).

El número de docentes de asignatura en la ENP es un dato interesante que es importante resaltar ya que, como se verá más adelante, la diferencia en número de personas en esta categoría es menor en comparación con el otro subsistema de bachillerato universitario que es el Colegio de Ciencias y Humanidades. Al mes de junio de 2019, de acuerdo con información de la RUPADGAPA, en la ENP los docentes de asignatura sumaban 1,830; de éstos 966 son mujeres y 864 son hombres; las primeras tienen un promedio de edad de 48 años, con una antigüedad promedio de 16 años de servicios, mientras que los hombres tienen un promedio de edad de 50 años y 17 años de servicios docentes. Las gráficas 10 y 11 nos muestran el dato porcentual de estas distribuciones.

\section{Profesores de asignatura en la ENP}

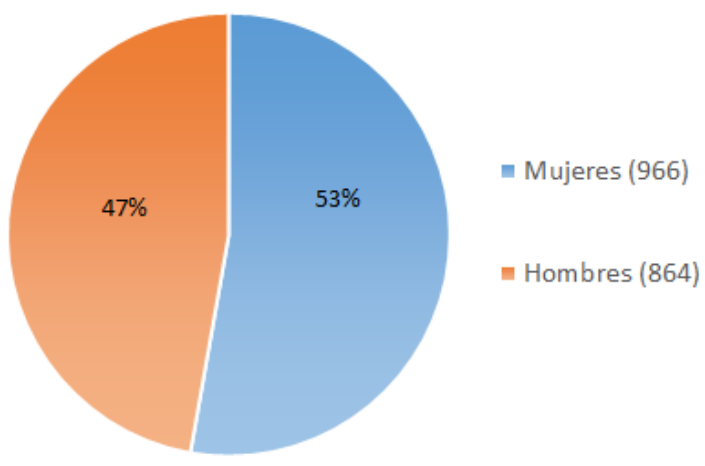

El nivel de estudios de los profesores de asignatura de la ENP es un dato relevante ya que de 1,830 personas que ostentan este nombramiento, tienen el grado de licenciatura 1,131 personas, cifra que representa más de 60\% de total de esta agrupación; 392 poseen el grado de maestro y es interesante que el tercer lugar lo ocupan los pasantes de licenciatura con 154 personas. 43 académicos en esta categoría tienen el grado de doctor, 11 cuentan con especialidad, hay 6 técnicos, y sólo 1 profesor de asignatura con posdoctorado. No se dispone información de 92 personas. En la gráfica 12 se muestra la distribución porcentual de las cifras. 
Gráfica 11. Edad y antigüedad de los profesores de asignatura de la ENP de la unAM.

Elaboración propia, con datos de RUPA-DGAPA, junio 2019.

Gráfica 12. Nivel de estudios de los profesores de asignatura de la ENP de la UNAM. Elaboración propia, con datos de RUPA-DGAPA, junio 2019.

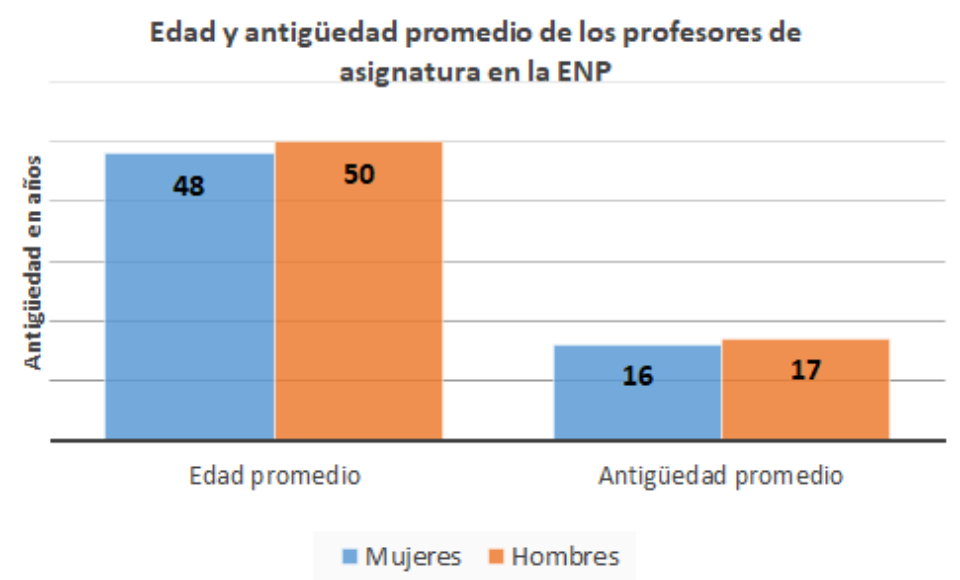

Nivel de estudios de los profesores de asigantura en la ENP

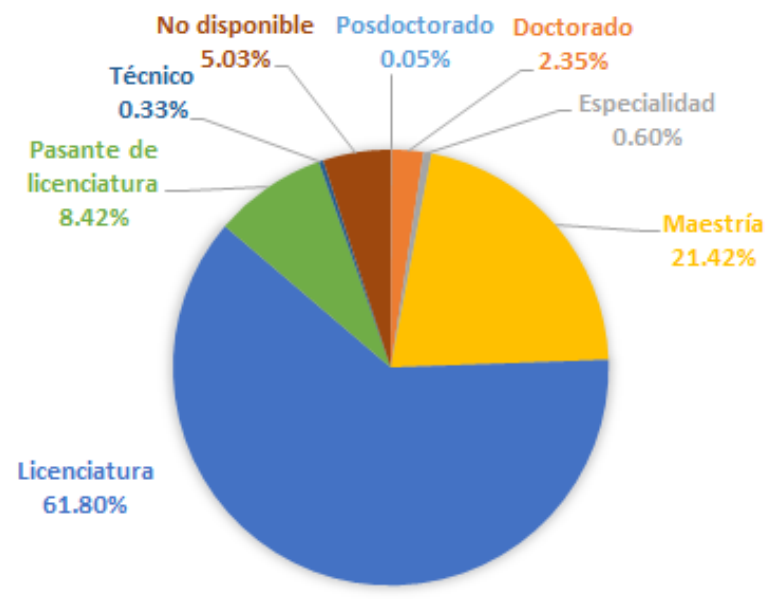

\section{Los profesores de asignatura en el Colegio de Ciencias y Humanidades}

Como se mencionó, la masificación de la educación media superior y superior de los años setenta trajo consigo la necesidad de crear espacios educativos para atender a una gran cantidad de jóvenes que demandaban un lugar para su formación. La unAm en 1971, consciente de ese crecimiento, crea uno de sus proyectos educativos más destacados para subsanar la urgencia: el ccH, en él se da cabida a jóvenes en la zona metropolitana de la Ciudad de México y su función es "resolver la desvinculación existente entre las diversas escuelas, facultades, institutos y centros de investigación de la unAM, así como para impulsar la transformación académica de la propia Universidad con una nueva perspectiva curricular y nuevos métodos de enseñanza" (ccH, s.f.). En sus inicios se inauguraron tres planteles, para 1972 se abrieron dos más, constituyéndose un subsistema de formación en el bachillerato que atiende a poco más de 58,000 estudiantes (UNAm, s.f.). Actualmente, el ccH es un sistema de formación 
Gráfica 13. Sexo de los profesores de asignatura del $\mathrm{CCH}$ de la unam. Elaboración propia, con datos de RUPA-DGAPA, junio 2019.

Gráfica 14. Edad y antigüedad de los profesores de asignatura del CCH de la UNAM.

Elaboración propia, con datos de RUPA-DGAPA, junio 2019. preuniversitaria que en el año de 1997 obtuvo el rango de Escuela Nacional y sus diversos planteles son coordinados por una Dirección General.

Los profesores de asignatura tienen un rol significativo ya que la atención al alumnado recae principalmente en los docentes con esta categoría académica; siguiendo con la información obtenida de la RUPA-DGAPA, en el mes de junio esta base de datos da cuenta de 2,391 personas en esta agrupación docente; de ellas 1,120 son mujeres y 1,271 son hombres. Las mujeres tienen una edad promedio de 46 años con una antigüedad docente de 14 años de servicios; en el caso de los hombres éstos tienen una edad promedio de 49 años contando con una antigüedad promedio de 16 años de actividad docente. Las gráficas 13 y 14 muestran la distribución porcentual de las cifras por sexo y las distribuciones por promedio de edad y años de servicios.

\section{Profesores de asignatura por sexo en el $\mathrm{CCH}$}

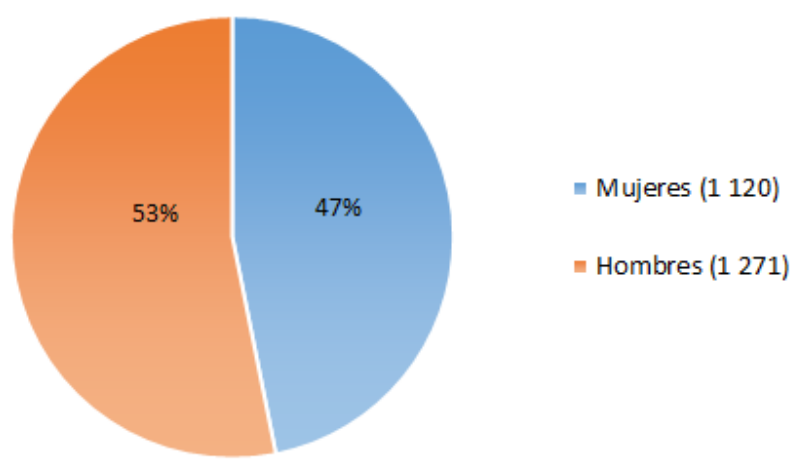

Edad y antigüedad promedio de los profesores de asignatura en el $\mathrm{CCH}$

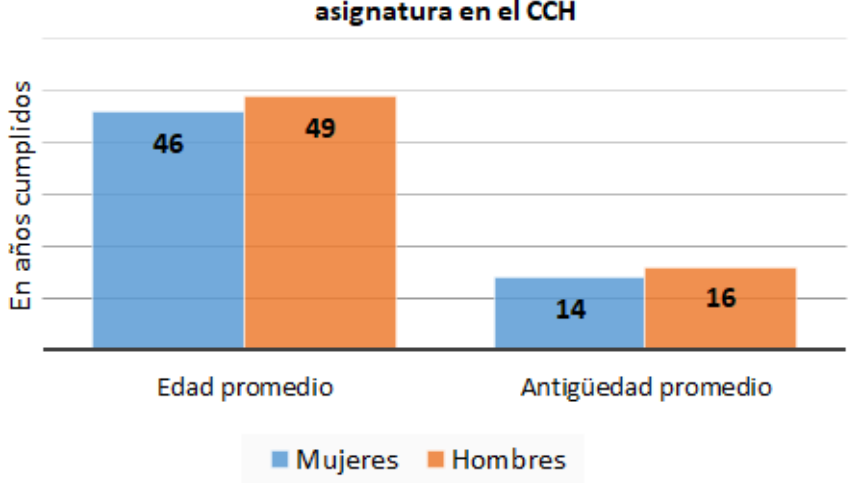

Al igual que en los análisis anteriores, presentamos información sobre la formación académica que poseen los profesores de asignatura en el ccH. De las 2,391 personas en esta categoría 1,600 tienen el grado de licenciatura, 553 el grado de maestría, 124 son pasantes de alguna licenciatura, 77 tienen el grado de doctor, 7 poseen una especialidad, sólo 1 persona tiene estudios de nivel técnico y no se posee información de 29. Es interesante señalar que ningún 
Gráfica 15. Nivel de estudios de los profesores de asignatura del CCH de la UnAm.

Elaboración propia, con datos de RUPA-DGAPA, junio 2019. profesor de asignatura tiene posdoctorado en este colectivo de docentes. En términos porcentuales, la gráfica 15 nos muestra la distribución de los datos.

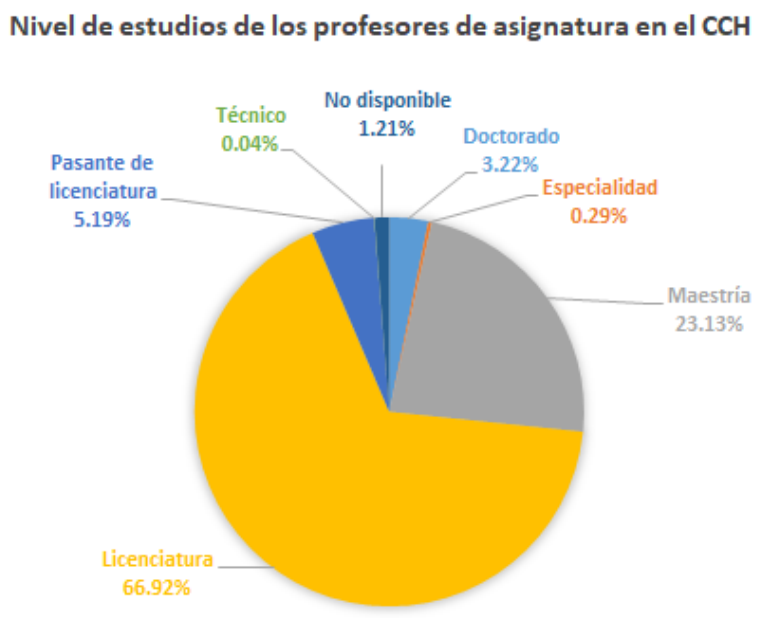

\section{Conclusiones}

Los docentes de asignatura son el colectivo más grande de la unAm. Como podemos observar en esta breve caracterización, entre aquellos que desempeñan la función docente en alguna de las agrupaciones aquí señaladas, sus características generales son muy similares entre sí; no obstante, hay algunos rasgos que, a modo de conclusión, es importante señalar.

La escolaridad predominante entre los profesores de asignatura en la ENP es de licenciatura, con 61.08\%, mientras que este mismo grado de estudios lo tiene el $66.92 \%$ del profesorado de asignatura en el $\mathrm{ccH}$. En las escuelas y facultades, $27.9 \%$ de los profesores tienen el grado de maestros y $13.62 \%$ el grado de doctor. En la comunidad de profesores de asignatura donde menos doctores hay es en la ENP, con tan sólo 2.35\%.

De esta manera, los profesores de asignatura representan la mayor cantidad de académicos en la Universidad y esta comunidad es la que posiblemente menos se ha estudiado para conocer sus necesidades, intereses, motivaciones y, porque no decirlo, sus obstáculos o dificultades en la realización de la tarea docente. Es por ello que este breve recuento pretende ser sólo una aproximación a lo mucho que debemos considerar para entender la dinámica de la función docente de los profesores de asignatura en la UNAM.

\section{Referencias}

* Asociación Autónoma del Personal Académico de la Universidad Nacional Autónoma de México (AAPaunam) (2019). Contrato Colectivo de Trabajo 2019-2021. Ciudad de México, México. Recuperado de http://www.aapaunam.mx/assets/cctaapaunam-2019-2021.pdf. 
* Colegio de Ciencias y Humanidades (ссн) (s.f.). Historia del Colegio de Ciencias y Humanidades. Recuperado de: https://www.cch.unam.mx/historia.

- Dirección General de la Escuela Nacional Preparatoria (s.f.). Acerca de la ENP. Recuperado de: http://dgenp.unam.mx/acercaenp/antecedentes.pdf.

* Martínez Hernández, A. M. P., Torres Carrasco, R., Hernández Romo, A. K., De Agüero Servín, M. M., Olvera López, A., Lara Gutiérrez, Y. A. y Pompa Mansilla, M. (2019). Diseño, desarrollo y análisis de los grupos focales para el proyecto de formación docente en la unam. En M. Sánchez y M. P. Martínez (Eds.), Formación Docente en la unam: Antecedentes y la voz de su profesorado (pp. 161-190). Ciudad de México, México: unam.

* Prellezo García, J. M. (coord.). (2009). Diccionario de Ciencias de la Educación. Madrid, España: ccs.

* Real Academia Española (2018). Diccionario de la lengua española, [23. ${ }^{\text {* ed., versión }}$ 23.2 en línea]. Recuperado de: https://dle.rae.es.

* Dirección General de Asuntos del Personal Académico de la unam. Registro Único del Personal Académico. Recuperado de: https://zafiro.dgapa.unam.mx/sipal usuario/opcion1:edadcol1/1.

* Universidad Nacional Autónoma de México (unam) (2019). Portal de Estadística Universitaria. Recuperado de: http://www.estadistica.unam.mx/numeralia/.

* Universidad Nacional Autónoma de México (unam) (s.f.). Agenda Estadística 2019 UNAM. Recuperado de: http://www.planeacion.unam.mx/Agenda/2019/disco/\#.

\section{Cómo citar este artículo}

* Torres Carrasco, Ruth (2019). ¿Cuál es la formación de la planta académica de la unAm? El caso de los profesores de asignatura. Revista Digital Universitaria (RDU). Vol. 20, núm. 6 noviembre-diciembre. Dol: http://doi.org/10.22201/ codeic.16076079e.2019.v20n6.a4.

Recepción: 28/10/2019. Aprobación: 31/10/2019 